\title{
Dynamic consistent NSFD scheme for a viral infection model with cellular infection and general nonlinear incidence
}

\author{
Jinhu $\mathrm{Xu}^{1 *}$, Jiangyong $\mathrm{Hou}^{2}$, Yan Geng ${ }^{3}$ and Suxia Zhang ${ }^{1}$
}

\section{"Correspondence:}

xujinhu09@163.com

'School of Sciences, Xi'an University

of Technology, Xi'an, P.R. China

Full list of author information is

available at the end of the article

\begin{abstract}
We study a discrete-time model with diffusion that describe the dynamics of viral infections by using nonstandard finite difference (NSFD) scheme. The original model we considered was a viral infection model with cellular infection and general nonlinear incidence. We analyze thoroughly the dynamical properties of both discrete and original continuous models and show that the discrete system is dynamically consistent with the original continuous model, including positivity and boundedness of solutions, equilibria, and their global properties. The results imply that the NSFD scheme can efficiently preserve the global dynamics properties of the corresponding continuous model. Some numerical simulations are carried out to validate the theoretical results.
\end{abstract}

Keywords: NSFD scheme; General nonlinear incidence; Discrete-time model; Global dynamics

\section{Introduction}

The classical within-host virus dynamics model is a system that includes three variables: uninfected cells $T(t)$, infected cells $I(t)$, and free virus particles $V(t)$ at time $t$ (see [1,2]). However, to take some features into consideration of a real system, such as delay between the moment of infection and the moment when the infected cell begins to produce the virus, additional classes of cells may be added to the system. For instance, for cells in the latent state an additional class, the class of exposed cells $W(t)$ has been introduced in [3], and the model reads as follows:

$$
\left\{\begin{array}{l}
T^{\prime}=\lambda-d T(t)-\beta_{1} T(t) V(t), \\
W^{\prime}=\beta_{1} X(t) V(t)-(\delta+\gamma) W(t), \\
I^{\prime}=\gamma W(t)-p I(t), \\
V^{\prime}=k I(t)-c V(t),
\end{array}\right.
$$

where $T(t), W(t), I(t)$, and $V(t)$ denote concentrations of uninfected cells, exposed cells, productively infected cells, and free virus particles at time $t$, respectively, $\lambda$ is the recruitment rate of the uninfected cells, $\beta_{1}$ is the virus-to-cell infection rate. $d, \delta, p$, and $c$ are the mortality rate of uninfected cells, exposed cells, infected cells, and free virus particles,

(c) The Author(s) 2018. This article is distributed under the terms of the Creative Commons Attribution 4.0 International License (http://creativecommons.org/licenses/by/4.0/), which permits unrestricted use, distribution, and reproduction in any medium, provided you give appropriate credit to the original author(s) and the source, provide a link to the Creative Commons license, and indicate if changes were made. 
respectively, and $1 / \gamma$ is the average time of the latent state. Free virus is produced from infected cells at the rate $k I$. The global dynamical behavior of model (1) has been studied in [3] by constructing Lyapunov functions.

Recent study shows that the interaction between pathogens and the immune response actually tends to be local within the body of infected hosts (see [4]). Hence, it is necessary to study the effect of spatial structure on virus dynamics, and much attention has been attracted by many researchers (see [5-9] and references therein). For example, Wang et al. [5] considered the following model taking the random mobility of viruses into account:

$$
\left\{\begin{array}{l}
\frac{\partial T}{\partial t}=\lambda-d T(x, t)-\beta_{1} T(x, t) V(x, t) \\
\frac{\partial I}{\partial t}=\beta_{1} T(x, t) V(x, t)-p I(x, t) \\
\frac{\partial V}{\partial t}=D \Delta V(x, t)+k I(x, t)-c V(x, t)
\end{array}\right.
$$

where $T(x, t), I(x, t)$, and $V(x, t)$ denote the densities of uninfected cells, infected cells, and free virus at position $x$ at time $t$, respectively, $D$ is the diffusion coefficient, and $\Delta$ is the Laplacian operator.

Notice that the studies mentioned only focus on virus-to-cell spread in the bloodstream. However, some works reveal that cell-to-cell infection is also vital to spread of virus in vivo (see [10-14]). Motivated by this fact, some models have been proposed to investigate the dynamics of within-host virus dynamics models to take both virus-to-cell and cell-to-cell infections into consideration (see [15-20] and references therein). However, the bilinear incidence rate is a simple description of the infection in the references mentioned. As mentioned in [21], a general incidence rate may help us to gain the unification theory by the omission of unessential details. Hence, inspired by the aforementioned work, we study the following model with general nonlinear incidence:

$$
\left\{\begin{array}{l}
\frac{\partial T}{\partial t}=\lambda-d T(t, x)-\beta_{1} T(x, t) f(V(x, t))-\beta_{2} T(x, t) g(I(x, t)), \\
\frac{\partial W}{\partial t}=\beta_{1} T(x, t) f(V(x, t))+\beta_{2} T(x, t) g(I(x, t))-(\delta+\gamma) W(x, t), \\
\frac{\partial I}{\partial t}=\gamma W(x, t)-p I(x, t), \\
\frac{\partial V}{\partial t}=D \Delta V(x, t)+k I(x, t)-c V(x, t) .
\end{array}\right.
$$

Here, $\beta_{2}$ is the cell-to-cell infection rate, and the other parameters have the same means as in system (1). The incidences are assumed to be the nonlinear responses to the concentrations of virus particles and infected cells, taking the forms $\beta_{1} T(x, t) f(V(x, t))$ and $\beta_{2} T(x, t) g(I(x, t))$, where $f$ and $g$ denote the forces of infection by virus particles and infected cells and satisfy the following properties (see [22, 23]):

$$
f(0)=g(0)=0, \quad f^{\prime}(V)>0, \quad g^{\prime}(I)>0, \quad f^{\prime \prime}(V) \leq 0, \quad g^{\prime \prime}(I) \leq 0 .
$$

It then follows from the mean value theorem that

$$
f^{\prime}(V) V \leq f(V) \leq f^{\prime}(0) V, \quad g^{\prime}(I) I \leq g(I) \leq g^{\prime}(0) I, \quad \text { for } I, V \geq 0 .
$$

Epidemiologically, condition (4) indicates that: (i) the disease cannot spread if there is no infection; (ii) the incidences $\beta_{1} T f(V)$ and $\beta_{2} T g(I)$ become faster as the densities of 
the virus particles and infected cells increase; (iii) the per capita infection rates by virus particles and infected cells will slow down due to certain inhibition effect since (5) implies that $\left(\frac{f(V)}{V}\right)^{\prime} \leq 0$ and $\left(\frac{g(I)}{I}\right)^{\prime} \leq 0$.

Obviously, the incidence rate with condition (4) contains the bilinear and saturation incidences. Thus, models (1) and (2) can be regarded as two particular cases of model (3). In this paper, we assume that model (3) is subject to the initial value conditions

$$
\begin{aligned}
& T(x, 0)=\psi_{1}(x) \geq 0, \quad W(x, 0)=\psi_{2}(x) \geq 0, \\
& I(x, 0)=\psi_{3}(x) \geq 0, \quad V(x, 0)=\psi_{4}(x) \geq 0, \quad x \in \bar{\Omega},
\end{aligned}
$$

and the Neumann boundary condition

$$
\frac{\partial V}{\partial \vec{n}}=0, \quad t>0, x \in \partial \Omega
$$

where $\Omega$ is a bounded domain in $\mathbb{R}^{n}$ with smooth boundary $\partial \Omega$, and $\frac{\partial}{\partial \vec{n}}$ is an outward normal vector of $\partial \Omega$.

It is worth noticing that discrete epidemic models have also been paid much attention by many researchers (see [24-26] and references therein). On one hand, discrete epidemic models have advantages in describing an infectious disease in comparison with continuous models since epidemic data are often collected at discrete times (such as daily, monthly, yearly, etc.). On the other hand, for some certain practical purposes, it is often necessary to obtain solutions of model (3) that describe the evolution of all variables with time. However, as we know, an exact analytical solution of differential equation system (3) is generally difficult or even impossible to be determined. Therefore, technical discretization is needed to obtain good analytical approximations of the solutions (see [27]). Meanwhile, we should keep in mind that the obtained discrete model should preserve the major dynamical properties of the original continuous model as much as possible. However, since a discrete model generally can exhibit more complicated dynamical behavior than continuous models such as bifurcations and chaos (see [25, 28] and references therein). Fortunately, a nonstandard finite difference scheme (NSFD) has been proposed by Mickens [29] and received much attention (see [20,30-38] and references therein). One important advantage of Mickens' scheme is that it performs well in preserving the major dynamical properties of the approximated original continuous models (see [20, 35-38]). More recently, Qin et al. [38] applied the NSFD scheme to discretized system (2) and showed that the discrete model has the same dynamics as the original system. Hence, following the idea of [29, 38], by applying the NSFD scheme to system (3) we obtain the following discrete system:

$$
\left\{\begin{array}{l}
\frac{T_{n+1}^{m}-T_{n}^{m}}{\Delta t}=\lambda-d T_{n+1}^{m}-\beta_{1} T_{n+1}^{m} f\left(V_{n}^{m}\right)-\beta_{2} T_{n+1}^{m} g\left(I_{n}^{m}\right), \\
\frac{W_{n+1}^{m}-W_{n}^{m}}{\Delta t}=\beta_{1} T_{n+1}^{m} f\left(V_{n}^{m}\right)+\beta_{2} T_{n+1}^{m} g\left(I_{n}^{m}\right)-(\delta+\gamma) W_{n+1}^{m}, \\
\frac{I_{n+1}^{m} I_{n}^{m}}{\Delta t}=\gamma W_{n+1}^{m}-p I_{n+1}^{m}, \\
\frac{V_{n+1}^{m}-V_{n}^{m}}{\Delta t}=D \frac{V_{n+1}^{m+1}-2 V_{n+1}^{m}+V_{n+1}^{m-1}}{(\Delta x)^{2}}+k I_{n+1}^{m}-c V_{n+1}^{m} .
\end{array}\right.
$$

Here we assume that $x \in \Omega=[a, b], \Delta t>0$ is the time step size, and $\Delta x=\frac{b-a}{N}$ is the space stepsize with positive integer $N$. Denote the mesh points as $\left\{\left(t_{n}, x_{m}\right), m=0,1,2, \ldots, N\right.$, 
$n \in \mathbb{N}\}$, where $t_{n}=n \Delta t$ and $x_{m}=a+m \Delta x$. Let $\left(T_{n}^{m}, W_{n}^{m}, I_{n}^{m}, V_{n}^{m}\right)$ be the approximations of the solution $\left(T\left(x_{m}, t_{n}\right), W\left(x_{m}, t_{n}\right), I\left(x_{m}, t_{n}\right), V\left(x_{m}, t_{n}\right)\right)$ of system (3) at each point. For convenience, we use an $(N+1)$-dimensional vector

$$
U_{n}=\left(U_{n}^{0}, U_{n}^{1}, \ldots, U_{n}^{N}\right)^{T}
$$

to represent the approximation $T, W, I$, or $V$ at time $t_{n}$, the notation $(\cdot)^{T}$ for the transposition of a vector, and the notation $U \geq 0$, meaning that all components of a vector $U$ are nonnegative. The discrete initial conditions take the form

$$
\begin{aligned}
& T_{0}^{m}=\psi_{1}\left(x_{m}\right) \geq 0, \quad W_{0}^{m}=\psi_{2}\left(x_{m}\right) \geq 0, \quad I_{0}^{m}=\psi_{3}\left(x_{m}\right) \geq 0, \\
& V_{0}^{m}=\psi_{4}\left(x_{m}\right) \geq 0 \quad \text { for } m \in\{0,1, \ldots, N\},
\end{aligned}
$$

and the discrete boundary conditions take the form

$$
V_{n}^{-1}=V_{n}^{0}, \quad V_{n}^{N}=V_{n}^{N+1} \quad \text { for } n \in \mathbb{N} .
$$

The goal of this paper is to show that the discrete system (8) by using Mickens' scheme can efficiently preserve the global asymptotic stability of the equilibria to the corresponding continuous system (3). The organization of this paper is as follows. In Sect. 2, we study the global dynamics of the continuous system (3). In Sect. 3, we investigate the global dynamics of the discrete system (8). It is then followed by numerical simulations in Sect. 4 . A brief conclusion ends the paper.

\section{Dynamics behavior of system (3)}

In this section, we study the threshold dynamics of the diffused system (3). First, we establish the global existence, positivity, and boundedness of solutions.

Theorem 2.1 For any given initial data satisfying condition (6), there exists a unique solution of problem (3)-(7) defined on $[0,+\infty)$, and this solution remains nonnegative and bounded for all $t>0$.

Proof The system can be written abstractly in the Banach space $X=C(\bar{\Omega}) \times C(\bar{\Omega})$ in the form

$$
\begin{aligned}
& U^{\prime}(t)=A U(t)+F(U(t)), \quad t>0, \\
& U(0)=U_{0} \in X,
\end{aligned}
$$

where $U=\operatorname{col}(T, W, I, V), U_{0}=\operatorname{col}\left(\psi_{1}, \psi_{2}, \psi_{3}, \psi_{4}\right), A U=\operatorname{col}(0,0,0, D \Delta V)$, and

$$
F(U(t))=\left(\begin{array}{c}
\lambda-d T(t, x)-\beta_{1} T(x, t) f(V(x, t))-\beta_{2} T(x, t) g(I(x, t)) \\
\beta_{1} T(x, t) f(V(x, t))+\beta_{2} T(x, t) g(I(x, t))-(\delta+\gamma) W(x, t) \\
\gamma W(x, t)-p I(x, t) \\
k I(x, t)-c V(x, t)
\end{array}\right) .
$$

It is clear that $F$ is locally Lipschitz in $X$. It follows from [39] that system (3) admits a unique local solution on $\left[0, T_{\max }\right)$, where $T_{\max }$ is the maximal existence time for solution 
of system (3). In addition, system (3) can be written in the form

$$
\begin{aligned}
& \frac{\partial T}{\partial t}=F_{1}(T, W, I, V), \\
& \frac{\partial W}{\partial t}=F_{2}(T, W, I, V), \\
& \frac{\partial I}{\partial t}=F_{3}(T, W, I, V), \\
& \frac{\partial V}{\partial t}-D \Delta V(x, t)=F_{4}(T, W, I, V) .
\end{aligned}
$$

It is easy to see that the functions $F_{i}(T, W, I, V), 1 \leq i \leq 4$, are continuously differentiable and satisfy the following conditions: $F_{1}(0, W, I, V)=\lambda \geq 0, F_{2}(T, 0, I, V)=$ $\beta_{1} T(x, t) f(V(x, t))+\beta_{2} T(x, t) g(I(x, t)) \geq 0, F_{3}(T, W, 0, V)=\gamma W \geq 0, F_{4}(T, W, I, 0)=k I \geq 0$ for all $T \geq 0, W \geq 0, I \geq 0, V \geq 0$. Since the initial data of system (3) are nonnegative, and from [40] we deduce the positivity of the local solution.

Next, we show the boundedness of solution. Let $G(x, t)=T(x, t)+W(x, t)+I(x, t)$. Then follows from system (3) that

$$
\begin{aligned}
\frac{\partial G(x, t)}{\partial t} & =\lambda-d T-\delta W-p I \\
& \leq \lambda-\mu G(x, t)
\end{aligned}
$$

where $\mu=\min \{d, \delta, p\}$. Then we have

$$
G(x, t) \leq G(x, 0) e^{-\mu t}+\frac{\lambda}{\mu}\left(1-e^{-\mu t}\right) .
$$

Thus, $T, W$, and $I$ are bounded. To proceed, it remains to prove the boundedness of $V$. From system (3) we have

$$
\left\{\begin{array}{l}
\frac{\partial V}{\partial t}-D \Delta V=k I-c V \\
\frac{\partial V}{\partial \vec{n}}=0 \\
V(x, 0)=\psi_{4}(x) \leq\left\|\psi_{4}\right\|_{\infty}=\max _{x \in \bar{\Omega}} \psi_{4}(x) .
\end{array}\right.
$$

By the comparison principle (see [41]) we obtain that $V(x, t) \leq \widetilde{V}(t)$, where $\widetilde{V}(t)=$ $\psi_{4}(x) e^{-c t}+\frac{k}{c}\|I\|\left(1-e^{-c t}\right)$ is the solution of the problem

$$
\left\{\begin{array}{l}
\frac{d \widetilde{V}}{d t}=k\|I\|-c \widetilde{V}, \\
\tilde{V}(0)=\left\|\psi_{4}\right\|_{\infty} .
\end{array}\right.
$$

Then the boundedness of $V$ is deduced from $\widetilde{V}(t) \leq \max \left\{\frac{k}{c}\|I\|,\left\|\psi_{4}\right\|_{\infty}\right.$ for $(x, t) \in \bar{\Omega} \times$ $\left.\left[0, T_{\max }\right)\right\}$. Therefore, we have proved that $T(x, t), W(x, t), I(x, t)$, and $V(x, t)$ are bounded on $\bar{\Omega} \times\left[0, T_{\max }\right)$. Thus, it follows from the standard theory for semilinear parabolic systems (see [42]) that $T_{\max }=+\infty$. This completes the proof. 
It is easy to see that system (3) has an infection-free steady state $E_{0}=\left(T_{0}, 0,0,0\right)$ with $T_{0}=\frac{\lambda}{d}$. This is the only biologically meaningful equilibrium if

$$
\mathfrak{R}_{0}=\frac{\lambda \gamma\left(\beta_{1} k f^{\prime}(0)+\beta_{2} c g^{\prime}(0)\right)}{d p c(\delta+\gamma)}<1
$$

which is the basic reproduction number of system (3). It then follows from system (3) that a positive steady state $E_{*}=\left(T_{*}, W_{*}, I_{*}, V_{*}\right)$ must satisfy the following equations:

$$
\left\{\begin{array}{l}
\lambda=d T+\beta_{1} T f(V)+\beta_{2} T g(I), \\
\beta_{1} T f(V)+\beta_{2} \operatorname{Tg}(I)=(\delta+\gamma) W \\
\gamma W=p I \\
k I=c V
\end{array}\right.
$$

Then we obtain that

$$
\lambda-d T=\frac{c p(\delta+\gamma) V}{k \gamma}, \quad W=\frac{p c V}{\gamma k}, \quad I=\frac{c V}{k} .
$$

This means that in order to have $T \geq 0, W, V>0$ at a steady state, we must have $V \in$ $\left(0, \frac{\lambda k \gamma}{p c(\delta+\gamma)}\right]$. Substitution of the second and third equations of (13) into the second equation of (12) gives

$$
T=\frac{(\delta+\gamma) p c V}{k \gamma\left(\beta_{1} f(V)+\beta_{2} g\left(\frac{c V}{k}\right)\right)} .
$$

Further, by substituting it into the first equation of (12) direct calculation yields

$$
0=\lambda-\frac{p c(\delta+\gamma) V}{k \gamma}-\frac{d p c(\delta+\gamma) V}{k \gamma\left(\beta_{1} f(V)+\beta_{2} g\left(\frac{c}{k} V\right)\right)}=: F(V) \text {. }
$$

From (5) we have $f(V)-V f^{\prime}(V) \geq 0$ and $g(I)-I g^{\prime}(I) \geq 0$. Thus, for all $V>0$, we obtain that

$$
F^{\prime}(V)=-\frac{(\delta+\gamma) p c}{k \gamma}-\frac{d p c(\delta+\gamma)\left[\beta_{1}\left(f(V)-V f^{\prime}(V)\right)+\beta_{2}\left(g\left(\frac{c V}{k}\right)-\frac{c}{k} g^{\prime}\left(\frac{c V}{k}\right)\right)\right]}{k \gamma\left(\beta_{1} f(V)+\beta_{2} g\left(\frac{c V}{k}\right)\right)^{2}}<0 .
$$

Furthermore, from (14) we have

$$
\begin{aligned}
& \lim _{V \rightarrow 0^{+}} F(V)=\frac{d p c(\delta+\gamma)\left(\Re_{0}-1\right)}{\gamma\left(\beta_{1} k f^{\prime}(0)+\beta_{2} c g^{\prime}(0)\right)}, \\
& F\left(\frac{\lambda k \gamma}{p c(\delta+\gamma)}\right)=-\frac{d p c(\delta+\gamma) V}{k \gamma\left(\beta_{1} f\left(\frac{\lambda k \gamma}{p c(\delta+\gamma)}\right)+\beta_{2} g\left(\frac{\lambda \gamma}{p(\delta+\gamma)}\right)\right)}<0 .
\end{aligned}
$$

It follows that an infection steady state $E_{*}=\left(T_{*}, W_{*}, I_{*}, V_{*}\right)$ exists when $\Re_{0}>1$.

Theorem 2.2 For system (3), we have:

(i) If $\Re_{0} \leq 1$, then there exists a unique infection-free equilibrium $E_{0}$;

(ii) If $\mathfrak{R}_{0}>1$, then there exists a unique infection equilibrium $E_{*}$ besides $E_{0}$. 


\subsection{Global stability analysis}

In this subsection, we establish the global asymptotic stability of the two steady states of system (3) by constructing Lyapunov functions.

Theorem 2.3 If $\mathfrak{R}_{0} \leq 1$, then the infection-free steady state $E_{0}$ is globally asymptotically stable.

Proof We construct a Lyapunov function as follows:

$$
L_{1}=\int_{\Omega}\left[T(x, t)-T_{0}-T_{0} \ln \frac{T(x, t)}{T_{0}}+W(x, t)+\frac{\delta+\gamma}{r} I(x, t)+\frac{\beta_{1} T_{0} f^{\prime}(0)}{\mathfrak{R}_{0} c} V(x, t)\right] d x .
$$

Calculating $\frac{d L_{1}}{d t}$ along the solutions of system (3) and applying $\lambda=d T_{0}$ and $\int_{\Omega} \Delta V(x, t) d x=$ 0 , we have

$$
\begin{aligned}
\frac{d L_{1}}{d t}= & \int_{\Omega}\left[\left(1-\frac{T_{0}}{T(x, t)}\right)\left(\lambda-d T(x, t)-\beta_{1} T(x, t) f(V(x, t))-\beta_{2} T(x, t) g(I(x, t))\right)\right. \\
& +\beta_{1} T(x, t) f(V(x, t))+\beta_{2} T(x, t) g(I(x, t))-(\delta+\gamma) W(x, t) \\
& \left.+\frac{\delta+\gamma}{\gamma}(\gamma W(x, t)-p I(x, t))+\frac{\beta_{1} T_{0} f^{\prime}(0)}{\mathfrak{R}_{0} c}(k I(x, t)-c V(x, t))\right] d x \\
= & \int_{\Omega}\left[d T_{0}\left(1-\frac{T_{0}}{T(x, t)}\right)\left(1-\frac{T(x, t)}{T_{0}}\right)+\beta_{1} T_{0} f(V(x, t))+\beta_{2} T_{0} g(I(x, t))\right. \\
& \left.-\frac{\beta_{1} T_{0} f^{\prime}(0)}{\mathfrak{R}_{0}} V(x, t)-\frac{\beta_{2} T_{0} g^{\prime}(0)}{\mathfrak{R}_{0}} I(x, t)\right] d x \\
\leq & \int_{\Omega}\left[d T_{0}\left(1-\frac{T_{0}}{T(x, t)}\right)\left(1-\frac{T(x, t)}{T_{0}}\right)+\frac{\beta_{1} T_{0} f^{\prime}(0)}{\mathfrak{R}_{0}}\left(\Re_{0}-1\right) V(x, t)\right. \\
& \left.+\frac{\beta_{2} T_{0} g^{\prime}(0)}{\mathfrak{R}_{0}}\left(\mathfrak{R}_{0}-1\right) I(x, t)\right] d x .
\end{aligned}
$$

Here, we used condition (5). Therefore, if $\mathfrak{R}_{0} \leq 1$, then $\frac{d L_{1}}{d t} \leq 0$. Furthermore, it can be shown that the largest invariant subset of $\left\{\frac{d L_{1}}{d t}=0\right\}$ is the singleton $\left\{E_{0}\right\}$. Using LaSalle's invariance principle, we derive that $E_{0}$ is globally asymptotically stable. This completes the proof.

Theorem 2.4 If $\Re_{0}>1$, then the infection steady state $E_{*}$ is globally asymptotically stable.

Proof We construct a Lyapunov function as follows:

$$
\begin{aligned}
L_{2}= & \int_{\Omega}\left[T(x, t)-T_{*}-T_{*} \ln \frac{T(x, t)}{T_{*}}+W(x, t)-W_{*}-W_{*} \ln \frac{W(x, t)}{W_{*}}\right. \\
& +\frac{\delta+\gamma}{\gamma}\left(I(x, t)-I_{*}-I_{*} \ln \frac{I(x, t)}{I_{*}}\right) \\
& \left.+\frac{\beta_{1} T_{*} f\left(V_{*}\right)}{k I_{*}}\left(V(x, t)-V_{*}-V_{*} \ln \frac{V(x, t)}{V_{*}}\right)\right] d x .
\end{aligned}
$$


Calculating the time derivative of $L_{2}$ along the trajectories of system (3), we obtain

$$
\begin{aligned}
\frac{d L_{2}}{d t}= & \int_{\Omega}\left[\left(1-\frac{T_{*}}{T(x, t)}\right)\left(\lambda-d T(x, t)-\beta_{1} T(x, t) f(V(x, t))-\beta_{2} T(x, t) g(I(x, t))\right)\right. \\
& +\left(1-\frac{W_{*}}{W(x, t)}\right)\left(\beta_{1} T(x, t) f(V(x, t))+\beta_{2} T(x, t) g(I(x, t))-(\delta+\gamma) W(x, t)\right) \\
& +\frac{\delta+\gamma}{\gamma}\left(1-\frac{I_{*}}{I(x, t)}\right)(\gamma W(x, t)-p I(x, t)) \\
& \left.+\frac{\beta_{1} T_{*} f\left(V_{*}\right)}{k I_{*}}\left(1-\frac{V_{*}}{V(x, t)}\right)(D \Delta V(x, t)+k I(x, t)-c V(x, t))\right] d x .
\end{aligned}
$$

Using the equilibrium conditions for $E_{*}$

$$
(\delta+\gamma) W_{*}=\beta_{1} T_{*} f\left(V_{*}\right)+\beta_{2} T_{*} g\left(I_{*}\right), \quad \gamma W_{*}=p I_{*}, \quad k I_{*}=c V_{*},
$$

we have

$$
\begin{aligned}
\frac{d L_{2}}{d t}= & \int_{\Omega}\left[d T_{*}\left(1-\frac{T_{*}}{T(x, t)}\right)\left(1-\frac{T(x, t)}{T_{*}}\right)+\left(1-\frac{T_{*}}{T(x, t)}\right)\right. \\
& \times\left(\beta_{1} T_{*} f\left(V_{*}\right)+\beta_{2} T_{*} g\left(I_{*}\right)-\beta_{1} T(x, t) f(V(x, t))-\beta_{2} T(x, t) g(I(x, t))\right) \\
& +\left(1-\frac{W_{*}}{W(x, t)}\right)\left(\beta_{1} T(x, t) f(V(x, t))+\beta_{2} T(x, t) g(I(x, t))\right. \\
& \left.-\left(\beta_{1} T_{*} f\left(V_{*}\right)+\beta_{2} T_{*} g\left(I_{*}\right)\right) \frac{W(x, t)}{W_{*}}\right)+\left(\beta_{1} T_{*} f\left(V_{*}\right)+\beta_{2} T_{*} g\left(I_{*}\right)\right) \\
& \times\left(1-\frac{I_{*}}{I(x, t)}\right)\left(\frac{W(x, t)}{W_{*}}-\frac{I(x, t)}{I_{*}}\right)+\beta_{1} T_{*} f\left(V_{*}\right)\left(1-\frac{V_{*}}{V(x, t)}\right) \\
& \left.\times\left(\frac{I(x, t)}{I_{*}}-\frac{V(x, t)}{V_{*}}\right)+\frac{\beta_{1} T_{*} f\left(V_{*}\right)}{k I_{*}} D \Delta V(x, t)\left(1-\frac{V_{*}}{V(x, t)}\right)\right] d x .
\end{aligned}
$$

Recalling that $\int_{\Omega} \Delta V(x, t) d x=0$ and $\int_{\Omega} \frac{\Delta V(x, t)}{V(x, t)} d x=\int_{\Omega} \frac{\|\nabla V(x, t)\|^{2}}{V^{2}(x, t)} d x$, we have

$$
\begin{aligned}
\frac{d L_{2}}{d t}= & \int_{\Omega}\left[d T_{*}\left(1-\frac{T_{*}}{T(x, t)}\right)\left(1-\frac{T(x, t)}{T_{*}}\right)+\beta_{1} T_{*} f\left(V_{*}\right)\left(4-\frac{T_{*}}{T(x, t)}\right.\right. \\
& \left.-\frac{I_{*} W(x, t)}{I(x, t) W_{*}}-\frac{T(x, t) f(V(x, t)) W_{*}}{T_{*} f\left(V_{*}\right) W(x, t)}-\frac{I(x, t) V_{*}}{I_{*} V(x, t)}+\frac{f(V(x, t))}{f\left(V_{*}\right)}-\frac{V(x, t)}{V_{*}}\right) \\
& +\beta_{2} T_{*} g\left(I_{*}\right)\left(3-\frac{T_{*}}{T(x, t)}-\frac{I_{*} W(x, t)}{I(x, t) W_{*}}-\frac{T(x, t) g(I(x, t)) W_{*}}{T_{*} g\left(I_{*}\right) W(x, t)}\right. \\
& \left.\left.+\frac{g(I(x, t))}{g\left(I_{*}\right)}-\frac{I(x, t)}{I_{*}}\right)\right] d x-\frac{\beta_{1} T_{*} f\left(V_{*}\right)}{k I_{*}} D V_{*} \int_{\Omega} \frac{\|\nabla V(x, t)\|^{2}}{V^{2}(x, t)} d x \\
= & \int_{\Omega}\left\{d T_{*}\left(1-\frac{T_{*}}{T(x, t)}\right)\left(1-\frac{T(x, t)}{T_{*}}\right)+\beta_{1} T_{*} f\left(V_{*}\right)\left[\varphi\left(\frac{T_{*}}{T(x, t)}\right)\right.\right. \\
& +\varphi\left(\frac{W(x, t) I_{*}}{W_{*} I(x, t)}\right)+\varphi\left(\frac{I(x, t) V_{*}}{I_{*} V(x, t)}\right)+\varphi\left(\frac{T(x, t) f(V(x, t)) W_{*}}{T_{*} f\left(V_{*}\right) W(x, t)}\right) \\
& \left.+\frac{f(V(x, t))}{f\left(V_{*}\right)}-\frac{V(x, t)}{V_{*}}+\ln \frac{f\left(V_{*}\right) V(x, t)}{f(V(x, t)) V_{*}}\right]+\beta_{2} T_{*} g\left(I_{*}\right)\left[\varphi\left(\frac{T_{*}}{T(x, t)}\right)\right.
\end{aligned}
$$




$$
\begin{aligned}
& +\varphi\left(\frac{I_{*} W(x, t)}{I(x, t) W_{*}}\right)+\varphi\left(\frac{T(x, t) g(I(x, t)) W_{*}}{T_{*} g\left(I_{*}\right) W(x, t)}\right)+\frac{g(I(x, t))}{g\left(I_{*}\right)}-\frac{I(x, t)}{I_{*}} \\
& \left.\left.+\ln \frac{g\left(I_{*}\right) I(x, t)}{g(I(x, t)) I_{*}}\right]\right\} d x-\frac{\beta_{1} T_{*} f\left(V_{*}\right)}{k I_{*}} D V_{*} \int_{\Omega} \frac{\|\nabla V(x, t)\|^{2}}{V^{2}(x, t)} d x \\
& =\int_{\Omega}\left\{d T_{*}\left(1-\frac{T_{*}}{T(x, t)}\right)\left(1-\frac{T(x, t)}{T_{*}}\right)+\beta_{1} T_{*} f\left(V_{*}\right)\left[\varphi\left(\frac{T_{*}}{T(x, t)}\right)\right.\right. \\
& +\varphi\left(\frac{W(x, t) I_{*}}{W_{*} I(x, t)}\right)+\varphi\left(\frac{I(x, t) V_{*}}{I_{*} V(x, t)}\right)+\varphi\left(\frac{T(x, t) f(V(x, t)) W_{*}}{T_{*} f\left(V_{*}\right) W(x, t)}\right) \\
& \left.+\varphi\left(\frac{f\left(V_{*}\right) V(x, t)}{f(V(x, t)) V_{*}}\right)+\left(\frac{f(V(x, t))}{f\left(V_{*}\right)}-\frac{V(x, t)}{V_{*}}\right)\left(1-\frac{f\left(V_{*}\right)}{f(V(x, t))}\right)\right] \\
& +\beta_{2} T_{*} g\left(I_{*}\right)\left[\varphi\left(\frac{T_{*}}{T(x, t)}\right)+\varphi\left(\frac{I_{*} W(x, t)}{I(x, t) W_{*}}\right)+\varphi\left(\frac{T(x, t) g(I(x, t)) W_{*}}{T_{*} g\left(I_{*}\right) W(x, t)}\right)\right. \\
& \left.\left.+\varphi\left(\frac{g\left(I_{*}\right) I(x, t)}{g(I(x, t)) I_{*}}\right)+\left(\frac{g(I(x, t))}{g\left(I_{*}\right)}-\frac{I(x, t)}{I_{*}}\right)\left(1-\frac{g\left(I_{*}\right)}{g(I(x, t))}\right)\right]\right\} d x \\
& +\frac{\beta_{1} T_{*} f\left(V_{*}\right)}{k I_{*}} D V_{*} \int_{\Omega} \frac{\|\nabla V(x, t)\|^{2}}{V^{2}(x, t)} d x,
\end{aligned}
$$

where $\varphi(x)=1+\ln x-x(x>0)$ which has a global maximum at $x=1$ and satisfies $\varphi(1)=0$. Moreover, from conditions (5) we easily obtain the following inequalities:

$$
\begin{aligned}
& \frac{f(V)}{f\left(V_{*}\right)} \geq \frac{V}{V_{*}} \quad \text { for } V \leq V_{*}, \quad \frac{f(V)}{f\left(V_{*}\right)} \leq \frac{V}{V_{*}} \quad \text { for } V \geq V_{*}, \\
& \frac{g(I)}{g\left(I_{*}\right)} \geq \frac{I}{I_{*}} \quad \text { for } I \leq I_{*}, \quad \frac{g(I)}{g\left(I_{*}\right)} \leq \frac{I}{I_{*}} \quad \text { for } I \geq I_{*},
\end{aligned}
$$

which imply that

$$
\left(\frac{f(V)}{f\left(V_{*}\right)}-\frac{V}{V_{*}}\right)\left(1-\frac{f\left(V_{*}\right)}{f(V)}\right) \leq 0, \quad\left(\frac{g(I)}{g\left(I_{*}\right)}-\frac{I}{I_{*}}\right)\left(1-\frac{g\left(I_{*}\right)}{g(I)}\right) \leq 0 .
$$

Then, it follows that $\frac{d L_{2}}{d t} \leq 0$, and $\frac{d L_{2}}{d t}=0$ if and only if $T=T_{*}, W=W_{*}, I=I_{*}$, and $V=V_{*}$. Hence, the largest invariant subset of $\left\{\frac{d L_{2}}{d t}=0\right\}$ is the singleton $\left\{E_{*}\right\}$. Thus, the global asymptotic stability of the infection steady state $E_{*}$ follows from LaSalle's invariance principle. This completes the proof.

\section{Dynamics behavior of system (8)}

The global asymptotic stability of the equilibria for the continuous system (3) have been obtained by constructing Lyapunov functions in Sect. 2. A natural question is whether the discrete system (8) can efficiently preserve the global asymptotic stability of the equilibria for corresponding continuous system (3). In this section, we deal with this problem.

It is easy to verify that system (8) has the same equilibria as system (3). We also denote the two equilibria as $E_{0}=\left(T_{0}, 0,0,0\right)$ and $E_{*}=\left(T_{*}, W_{*}, I_{*}, V_{*}\right)$. Rearranging the formulations 
in equations of (8) yields

$$
\left\{\begin{array}{l}
T_{n+1}^{m}=\frac{\lambda \Delta t+T_{n}^{m}}{1+\Delta t\left(d+\beta_{1} f\left(V_{n}^{m}\right)+\beta_{2} g\left(I_{n}^{m}\right)\right)}, \\
W_{n+1}^{m}=\frac{W_{n}^{m}+\Delta t\left(\beta_{1} T_{n+1}^{m} f\left(V_{n}^{m}\right)+\beta_{2} T_{n+1}^{m} g\left(I_{n}^{m}\right)\right)}{1+\Delta t(\delta+\gamma)} \\
I_{n+1}^{m}=\frac{I_{n}^{m}+\Delta t \gamma W_{n+1}^{m}}{1+\Delta t p} \\
A V_{n+1}=V_{n}+k \Delta t I_{n+1},
\end{array}\right.
$$

where the square matrix $A$ of dimension $(N+1) \times(N+1)$ is given by

$$
\left(\begin{array}{ccccccc}
c_{1} & c_{2} & 0 & \cdots & 0 & 0 & 0 \\
c_{2} & c_{3} & c_{2} & \cdots & 0 & 0 & 0 \\
0 & c_{2} & c_{3} & \cdots & 0 & 0 & 0 \\
\vdots & \vdots & \vdots & \ddots & \vdots & \vdots & \vdots \\
0 & 0 & 0 & \cdots & c_{3} & c_{2} & 0 \\
0 & 0 & 0 & \cdots & c_{2} & c_{3} & c_{2} \\
0 & 0 & 0 & \cdots & 0 & c_{2} & c_{1}
\end{array}\right)
$$

with $c_{1}=1+D \Delta t /(\Delta x)^{2}+c \Delta t, c_{2}=-D \Delta t /(\Delta x)^{2}$, and $c_{3}=1+2 D \Delta t /(\Delta x)^{2}+c \Delta t$. Hence, $A$ is nonsingular, and the last equation of system (15) is equivalent to

$$
V_{n+1}=A^{-1}\left(V_{n}+k \Delta t I_{n+1}\right)
$$

Since all parameters in (8) are positive, it is clear that if the initial values satisfy $T_{n} \geq 0$, $W_{n} \geq 0, I_{n} \geq 0, V_{n} \geq 0$, then the solution remains positive for all $m \geq 0$ by mathematical induction. Therefore, we can obtain the following result.

Theorem 3.1 Given $\Delta t>0$ and $\Delta x>0$, the solutions of system (8) satisfy $T_{n} \geq 0, W_{n} \geq 0$, $I_{n} \geq 0, V_{n} \geq 0$ for all $n \in \mathbb{N}$.

\subsection{Global stability}

In this subsection, we establish the global stability of the infection-free steady state and the infection steady state of system (8) by constructing discrete Lyapunov functions.

Theorem 3.2 Given $\Delta t>0$ and $\Delta x>0$, if $\mathfrak{R}_{0} \leq 1$, then the infection-free equilibrium $E_{0}$ of system (8) is globally asymptotically stable.

Proof Define a discrete Lyapunov function

$$
\begin{aligned}
G_{n}= & \sum_{m=0}^{N} \frac{1}{\Delta t}\left[T_{n}^{m}-T_{0}-T_{0} \ln \frac{T_{n}^{m}}{T_{0}}+W_{n}^{m}+\frac{\delta+\gamma}{\gamma}\left(1+\frac{\gamma}{\delta+\gamma} \frac{\beta_{2} T_{0} g^{\prime}(0)}{\mathfrak{R}_{0}} \Delta t\right) I_{n}^{m}\right. \\
& \left.+\frac{\beta_{1} T_{0} f^{\prime}(0)}{c \Re_{0}}(1+c \Delta t) V_{n}^{m}\right] .
\end{aligned}
$$

Since $x-1 \geq \ln x$ for all $x>0$, it is clear that $G_{n} \geq 0$ for all $n \in \mathbb{N}$. Then we have

$$
G_{n+1}-G_{n}=\sum_{m=0}^{N} \frac{1}{\Delta t}\left[T_{n+1}^{m}-T_{n}^{m}+T_{0} \ln \frac{T_{n}^{m}}{T_{n+1}^{m}}+W_{n+1}^{m}-W_{n}^{m}\right.
$$




$$
\begin{aligned}
& +\frac{\delta+\gamma}{\gamma}\left(1+\frac{\gamma}{\delta+\gamma} \frac{\beta_{2} T_{0} g^{\prime}(0)}{\mathfrak{R}_{0}} \Delta t\right) \times\left(I_{n+1}^{m}-I_{n}^{m}\right) \\
& \left.+\frac{\beta_{1} T_{0} f^{\prime}(0)}{c \mathfrak{R}_{0}}(1+c \Delta t)\left(V_{n+1}^{m}-V_{n}^{m}\right)\right] \\
\leq & \sum_{m=0}^{N} \frac{1}{\Delta t}\left[\left(1-\frac{T_{0}}{T_{n+1}^{m}}\right)\left(T_{n+1}^{m}-T_{n}^{m}\right)+W_{n+1}^{m}-W_{n}^{m}\right. \\
& +\frac{\delta+\gamma}{\gamma}\left(1+\frac{\gamma}{\delta+\gamma} \frac{\beta_{2} T_{0} g^{\prime}(0)}{\mathfrak{R}_{0}} \Delta t\right) \times\left(I_{n+1}^{m}-I_{n}^{m}\right) \\
& \left.+\frac{\beta_{1} T_{0} f^{\prime}(0)}{c \mathfrak{R}_{0}}(1+c \Delta t)\left(V_{n+1}^{m}-V_{n}^{m}\right)\right] \\
= & \sum_{m=0}^{N}\left[\left(1-\frac{T_{0}}{T_{n+1}^{m}}\right)\left(d T_{0}-d T_{n+1}^{m}-\beta_{1} T_{n+1}^{m} f\left(V_{n}^{m}\right)-\beta_{2} T_{n+1}^{m} g\left(I_{n}^{m}\right)\right)\right. \\
& +\beta_{1} T_{n+1}^{m} f\left(V_{n}^{m}\right)+\beta_{2} T_{n+1}^{m} g\left(I_{n}^{m}\right)-(\delta+\gamma) W_{n+1}^{m}+\frac{\delta+\gamma}{\gamma}\left(\gamma W_{n+1}^{m}-p I_{n+1}^{m}\right) \\
& +\frac{\beta_{2} T_{0} g^{\prime}(0)}{\mathfrak{R}_{0}}\left(I_{n+1}^{m}-I_{n}^{m}\right)+\frac{\beta_{1} T_{0} f^{\prime}(0)}{c \Re_{0}}\left(k I_{n+1}^{m}-c V_{n+1}^{m}\right) \\
& \left.+\frac{\beta_{1} T_{0} f^{\prime}(0)}{\mathfrak{R}_{0}}\left(V_{n+1}^{m}-V_{n}^{m}\right)+\frac{\beta_{1} T_{0} f^{\prime}(0) D}{c \Re_{0}(\Delta x)^{2}}\left(V_{n+1}^{m+1}-2 V_{n+1}^{m}+V_{n+1}^{m-1}\right)\right] \\
& N \\
\leq & \sum_{m=0}^{N}\left[d T_{0}\left(1-\frac{T_{0}}{T_{n+1}^{m}}\right)\left(1-\frac{T_{n+1}^{m}}{T_{0}}\right)+\frac{\beta_{1} T_{0} f^{\prime}(0)}{\mathfrak{R}_{0}}\left(\Re_{0}-1\right) V_{n}^{m}\right. \\
& \left.+\frac{\beta_{2} T_{0} g^{\prime}(0)}{\mathfrak{R}_{0}}\left(\Re_{0}-1\right) I_{n}^{m}\right]+\frac{\beta_{1} T_{0} f^{\prime}(0) D}{c \Re_{0}(\Delta x)^{2}}\left(V_{n+1}^{N+1}-V_{n+1}^{N}+V_{n+1}^{-1}-V_{n+1}^{0}\right) \\
= & \sum_{m=0}^{N}\left[d T_{0}\left(1-\frac{T_{0}}{T_{n+1}^{m}}\right)\left(1-\frac{T_{n+1}^{m}}{T_{0}}\right)+\frac{\beta_{1} T_{0} f^{\prime}(0)}{\mathfrak{R}_{0}}\left(\mathfrak{R}_{0}-1\right) V_{n}^{m}\right. \\
& \left.\frac{\beta_{2} T_{0} g^{\prime}(0)}{\Re_{0}}\left(\Re_{0}-1\right) I_{n}^{m}\right] . \\
&
\end{aligned}
$$

It then follows that if $\Re_{0} \leq 1$, then

$$
G_{n+1}-G_{n} \leq 0
$$

for all $n \in \mathbb{N}$, which implies that $G_{n}$ is a decreasing sequence. Since $G_{n} \geq 0$, there is a limit $\lim _{n \rightarrow \infty} G_{n} \geq 0$, which yields $\lim _{n \rightarrow \infty}\left(G_{n+1}-G_{n}\right)=0$. Thus, we have:

(1) If $\Re_{0}<1$, then it follows from $\lim _{n \rightarrow \infty}\left(G_{n+1}-G_{n}\right)=0$ that $\lim _{n \rightarrow \infty} T_{n}^{m}=T_{0}$ and $\lim _{n \rightarrow \infty} I_{n}^{m}=\lim _{n \rightarrow \infty} V_{n}^{m}=0$. Further, from system (8) we have $\lim _{n \rightarrow \infty} W_{n}^{m}=0$.

(2) If $\Re_{0}=1$, then it follows from $\lim _{n \rightarrow \infty}\left(G_{n+1}-G_{n}\right)=0$ that $\lim _{n \rightarrow \infty} T_{n}^{m}=T_{0}$. By the first equation of system (8) we obtain that $\beta_{1} T_{0} f\left(V_{n}^{m}\right)+\beta_{2} T_{0} g\left(I_{n}^{m}\right)=0$. Since $T_{0}>0$, then from (4) we have $I_{n}^{m}=V_{n}^{m}=0$. Furthermore, from the second equation of system (8) we obtain that $W_{n}^{m}=0$.

Thus, we conclude that if $\mathfrak{R}_{0} \leq 1$, then $E_{0}$ is globally asymptotically stable. This completes the proof.

Theorem 3.3 Given $\Delta t>0$ and $\Delta x>0$, the infection equilibrium $E_{*}$ of system (8) is globally asymptotically stable when $\mathfrak{R}_{0}>1$. 
Proof Define a discrete Lyapunov function

$$
\begin{aligned}
\widetilde{G}_{n}= & \sum_{m=0}^{N} \frac{1}{\Delta t}\left\{T_{n}^{m}-T_{*}-T_{*} \ln \frac{T_{n}^{m}}{T_{*}}+W_{n}^{m}-W_{*}-W_{*} \ln \frac{W_{n}^{m}}{W_{*}}\right. \\
& +\frac{\delta+\gamma}{\gamma}\left(I_{n}^{m}-I_{*}-I_{*} \ln \frac{I_{n}^{m}}{I_{*}}\right)+\frac{\beta_{1} T_{*} f\left(V_{*}\right)}{k I_{*}}\left(V_{n}^{m}-V_{*}-V_{*} \ln \frac{V_{n}^{m}}{V_{*}}\right) \\
& \left.+\Delta t \beta_{1} T_{*} f\left(V_{*}\right) \varphi\left(\frac{f\left(V_{n}^{m}\right)}{f\left(V_{*}\right)}\right)+\Delta t \beta_{2} T_{*} g\left(I_{*}\right) \varphi\left(\frac{g\left(I_{n}^{m}\right)}{g\left(I_{*}\right)}\right)\right\},
\end{aligned}
$$

where $\varphi(x)=x-1-\ln x(x>0)$ has a global minimum at $x=1$ and satisfies $\varphi(1)=0$.

The difference of $\widetilde{G}_{n}$ satisfies

$$
\begin{aligned}
& \widetilde{G}_{n+1}-\widetilde{G}_{n}=\sum_{m=0}^{N} \frac{1}{\Delta t}\left\{T_{n+1}^{m}-T_{n}^{m}+T_{*} \ln \frac{T_{n}^{m}}{T_{n+1}^{m}}+W_{n+1}^{m}-W_{n}^{m}+W_{*} \ln \frac{W_{n}^{m}}{W_{n+1}^{m}}\right. \\
& +\frac{\delta+\gamma}{\gamma}\left(I_{n+1}^{m}-I_{n}^{m}+I_{*} \ln \frac{I_{n}^{m}}{I_{n+1}^{m}}\right)+\frac{\beta_{1} T_{*} f\left(V_{*}\right)}{k I_{*}}\left(V_{n+1}^{m}-V_{n}^{m}\right. \\
& \left.+V_{*} \ln \frac{V_{n}^{m}}{V_{n+1}^{m}}\right)+\Delta t \beta_{1} T_{*} f\left(V_{*}\right)\left(\frac{f\left(V_{n+1}^{m}\right)}{f\left(V_{*}\right)}-\frac{f\left(V_{n}^{m}\right)}{f\left(V_{*}\right)}+\ln \frac{f\left(V_{n}^{m}\right)}{f\left(V_{n+1}^{m}\right)}\right) \\
& \left.+\Delta t \beta_{2} T_{*} g\left(I_{*}\right)\left(\frac{g\left(I_{n+1}^{m}\right)}{g\left(I_{*}\right)}-\frac{g\left(I_{n}^{m}\right)}{g\left(I_{*}\right)}+\ln \frac{g\left(I_{n}^{m}\right)}{g\left(I_{n+1}^{m}\right)}\right)\right\} \\
& \leq \sum_{m=0}^{N} \frac{1}{\Delta t}\left\{\left(1-\frac{T_{*}}{T_{n+1}^{m}}\right)\left(T_{n+1}^{m}-T_{n}^{m}\right)+\left(1-\frac{W_{*}}{W_{n+1}^{m}}\right)\left(W_{n+1}^{m}-W_{n}^{m}\right)\right. \\
& +\frac{\delta+\gamma}{\gamma}\left(1-\frac{I_{*}}{I_{n+1}^{m}}\right)\left(I_{n+1}^{m}-I_{n}^{m}\right)+\frac{\beta_{1} T_{*} f\left(V_{*}\right)}{k I_{*}}\left(1-\frac{V_{*}}{V_{n+1}^{m}}\right) \\
& \times\left(V_{n+1}^{m}-V_{n}^{m}\right)+\Delta t \beta_{1} T_{*} f\left(V_{*}\right)\left(\frac{f\left(V_{n+1}^{m}\right)}{f\left(V_{*}\right)}-\frac{f\left(V_{n}^{m}\right)}{f\left(V_{*}\right)}+\ln \frac{f\left(V_{n}^{m}\right)}{f\left(V_{n+1}^{m}\right)}\right) \\
& \left.+\Delta t \beta_{2} T_{*} g\left(I_{*}\right)\left(\frac{g\left(I_{n+1}^{m}\right)}{g\left(I_{*}\right)}-\frac{g\left(I_{n}^{m}\right)}{g\left(I_{*}\right)}+\ln \frac{g\left(I_{n}^{m}\right)}{g\left(I_{n+1}^{m}\right)}\right)\right\} \\
& =\sum_{m=0}^{N}\left\{\left(1-\frac{T_{*}}{T_{n+1}^{m}}\right)\left(\lambda-d T_{n+1}^{m}-\beta_{1} T_{n+1}^{m} f\left(V_{n}^{m}\right)-\beta_{2} T_{n+1}^{m} g\left(I_{n}^{m}\right)\right)\right. \\
& +\left(1-\frac{W_{*}}{W_{n+1}^{m}}\right)\left(\beta_{1} T_{n+1}^{m} f\left(V_{n}^{m}\right)+\beta_{2} T_{n+1}^{m} g\left(I_{n}^{m}\right)-(\delta+\gamma) W_{n+1}^{m}\right) \\
& +\frac{\delta+\gamma}{\gamma}\left(1-\frac{I_{*}}{I_{n+1}^{m}}\right)\left(\gamma W_{n+1}^{m}-p I_{n+1}^{m}\right) \\
& +\frac{\beta_{1} T_{*} f\left(V_{*}\right)}{k I_{*}}\left(1-\frac{V_{*}}{V_{n+1}^{m}}\right)\left(D \frac{V_{n+1}^{m+1}-2 V_{n+1}^{m}+V_{n+1}^{m-1}}{(\Delta x)^{2}}+k I_{n+1}^{m}-c V_{n+1}^{m}\right) \\
& +\beta_{1} T_{*} f\left(V_{*}\right)\left(\frac{f\left(V_{n+1}^{m}\right)}{f\left(V_{*}\right)}-\frac{f\left(V_{n}^{m}\right)}{f\left(V_{*}\right)}+\ln \frac{f\left(V_{n}^{m}\right)}{f\left(V_{n+1}^{m}\right)}\right) \\
& \left.+\beta_{2} T_{*} g\left(I_{*}\right)\left(\frac{g\left(I_{n+1}^{m}\right)}{g\left(I_{*}\right)}-\frac{g\left(I_{n}^{m}\right)}{g\left(I_{*}\right)}+\ln \frac{g\left(I_{n}^{m}\right)}{g\left(I_{n+1}^{m}\right)}\right)\right\} .
\end{aligned}
$$


Using the equilibrium condition (12) for $E_{*}$, we obtain that

$$
\begin{aligned}
& \widetilde{G}_{n+1}-\widetilde{G}_{n} \leq \sum_{m=0}^{N}\left\{d T_{*}\left(1-\frac{T_{*}}{T_{n+1}^{m}}\right)\left(1-\frac{T_{n+1}^{m}}{T_{*}}\right)+\left(\beta_{1} T_{*} f\left(V_{*}\right)+\beta_{2} T_{*} g\left(I_{*}\right)\right)\right. \\
& \times\left(1-\frac{T_{*}}{T_{n+1}^{m}}\right)+\left(\beta_{1} T_{n+1}^{m} f\left(V_{n}^{m}\right)+\beta_{2} T_{n+1}^{m} g\left(I_{n}^{m}\right)\right)\left(\frac{T_{*}}{T_{n+1}^{m}}-1\right) \\
& +\left(\beta_{1} T_{n+1}^{m} f\left(V_{n}^{m}\right)+\beta_{2} T_{n+1}^{m} g\left(I_{n}^{m}\right)\right)\left(1-\frac{W_{*}}{W_{n+1}^{m}}\right) \\
& +\left(\beta_{1} T_{*} f\left(V_{*}\right)+\beta_{2} T_{*} g\left(I_{*}\right)\right)\left(1-\frac{W_{n+1}^{m}}{W_{*}}\right) \\
& +\left(\beta_{1} T_{*} f\left(V_{*}\right)+\beta_{2} T_{*} g\left(I_{*}\right)\right)\left(1-\frac{I_{*}}{I_{n+1}^{m}}\right)\left(\frac{W_{n+1}^{m}}{W_{*}}-\frac{I_{n+1}^{m}}{I_{*}}\right) \\
& +\beta_{1} T_{*} f\left(V_{*}\right)\left(1-\frac{V_{*}}{V_{n+1}^{m}}\right)\left(\frac{I_{n+1}^{m}}{I_{*}}-\frac{V_{n+1}^{m}}{V_{*}}\right) \\
& +\beta_{1} T_{*} f\left(V_{*}\right)\left(\frac{f\left(V_{n+1}^{m}\right)}{f\left(V_{*}\right)}-\frac{f\left(V_{n}^{m}\right)}{f\left(V_{*}\right)}+\ln \frac{f\left(V_{n}^{m}\right)}{f\left(V_{n+1}^{m}\right)}\right) \\
& \left.+\beta_{2} T_{*} g\left(I_{*}\right)\left(\frac{g\left(I_{n+1}^{m}\right)}{g\left(I_{*}\right)}-\frac{g\left(I_{n}^{m}\right)}{g\left(I_{*}\right)}+\ln \frac{g\left(I_{n}^{m}\right)}{g\left(I_{n+1}^{m}\right)}\right)\right\} \\
& +\sum_{m=0}^{N} \frac{D \beta_{1} T_{*} f\left(V_{*}\right)}{k I_{*}(\Delta x)^{2}}\left(1-\frac{V_{*}}{V_{n+1}^{m}}\right)\left(V_{n+1}^{m+1}-2 V_{n+1}^{m}+V_{n+1}^{m-1}\right) \\
& =\sum_{m=0}^{N}\left\{d T_{*}\left(1-\frac{T_{*}}{T_{n+1}^{m}}\right)\left(1-\frac{T_{n+1}^{m}}{T_{*}}\right)+\beta_{2} T_{*} f\left(V_{*}\right)\left[4-\frac{T_{*}}{T_{n+1}}\right.\right. \\
& -\frac{T_{n+1} f\left(V_{n}^{m}\right) W_{*}}{T_{*} f\left(V_{*}\right) W_{n+1}^{m}}-\frac{I_{*} W_{n+1}^{m}}{I_{n+1}^{m} W_{*}}-\frac{V_{*} I_{n+1}^{m}}{V_{n+1}^{m} I_{*}}+\frac{f\left(V_{n+1}^{m}\right)}{f\left(V_{*}\right)}-\frac{V_{n+1}^{m}}{V_{*}} \\
& \left.+\ln \frac{f\left(V_{n}^{m}\right)}{f\left(V_{n+1}^{m}\right)}\right]+\beta_{2} T_{*} g\left(I_{*}\right)\left[3-\frac{T_{*}}{T_{n+1}^{m}}-\frac{T_{n+1}^{m} g\left(I_{n}^{m}\right) W_{*}}{T_{*} g\left(I_{*}\right) W_{n+1}^{m}}\right. \\
& \left.\left.-\frac{I_{*} W_{n+1}^{m}}{I_{n+1}^{m} W_{*}}+\frac{g\left(I_{n+1}^{m}\right)}{g\left(I_{*}\right)}-\frac{I_{n+1}^{m}}{I_{*}}+\ln \frac{g\left(I_{n}^{m}\right)}{g\left(I_{n+1}^{m}\right)}\right]\right\} \\
& -\sum_{m=0}^{N-1} \frac{D V_{*} \beta_{1} T_{*} f\left(V_{*}\right)}{k I_{*}(\Delta x)^{2}} \frac{\left(V_{n+1}^{m+1}-V_{n+1}^{m}\right)^{2}}{V_{n+1}^{m+1} V_{n+1}^{m}} \\
& =\sum_{m=0}^{N}\left\{d T_{*}\left(1-\frac{T_{*}}{T_{n+1}^{m}}\right)\left(1-\frac{T_{n+1}^{m}}{T_{*}}\right)+\beta_{1} T_{*} f\left(V_{*}\right)\left[-\varphi\left(\frac{T_{*}}{T_{n+1}^{m}}\right)\right.\right. \\
& -\varphi\left(\frac{T_{n+1}^{m} f\left(V_{n}^{m}\right) W_{*}}{T_{*} f\left(V_{*}\right) W_{n+1}^{m}}\right)-\varphi\left(\frac{I_{*} W_{n+1}^{m}}{I_{n+1}^{m} W *}\right)-\varphi\left(\frac{V_{*} I_{n+1}^{m}}{V_{n+1}^{m} I_{*}}\right)+\frac{f\left(V_{n+1}^{m}\right)}{f\left(V_{*}\right)} \\
& \left.-\frac{V_{n+1}^{m}}{V_{*}}+\ln \frac{f\left(V_{*}\right) V_{n+1}^{m}}{f\left(V_{n+1}^{m}\right) V_{*}}\right]+\beta_{2} T_{*} g\left(I_{*}\right)\left[-\varphi\left(\frac{T_{*}}{T_{n+1}^{m}}\right)-\varphi\left(\frac{I_{*} W_{n+1}^{m}}{I_{n+1}^{m} W_{*}}\right)\right. \\
& \left.\left.-\varphi\left(\frac{T_{n+1}^{m} g\left(I_{n}^{m}\right) W_{*}}{T_{*} g\left(I_{*}\right) W_{n+1}^{m}}\right)+\frac{g\left(I_{n+1}^{m}\right)}{g\left(I_{*}\right)}-\frac{I_{n+1}^{m}}{I_{*}}+\ln \frac{g\left(I_{*}\right) I_{n+1}^{m}}{g\left(I_{n+1}^{m}\right) I_{n+1}^{m}}\right]\right\} \\
& -\sum_{m=0}^{N-1} \frac{D V_{*} \beta_{1} T_{*} f\left(V_{*}\right)}{k I_{*}(\Delta x)^{2}} \frac{\left(V_{n+1}^{m+1}-V_{n+1}^{m}\right)^{2}}{V_{n+1}^{m+1} V_{n+1}^{m}}
\end{aligned}
$$




$$
\begin{aligned}
= & \sum_{m=0}^{N}\left\{d T_{*}\left(1-\frac{T_{*}}{T_{n+1}^{m}}\right)\left(1-\frac{T_{n+1}^{m}}{T_{*}}\right)+\beta_{1} T_{*} f\left(V_{*}\right)\left[-\varphi\left(\frac{T_{*}}{T_{n+1}^{m}}\right)\right.\right. \\
& -\varphi\left(\frac{T_{n+1}^{m} f\left(V_{n}^{m}\right) W_{*}}{T_{*} f\left(V_{*}\right) W_{n+1}^{m}}\right)-\varphi\left(\frac{I_{*} W_{n+1}^{m}}{I_{n+1}^{m} W *}\right)-\varphi\left(\frac{V_{*} I_{n+1}^{m}}{V_{n+1}^{m} I_{*}}\right) \\
& \left.-\varphi\left(\frac{f\left(V_{*}\right) V_{n+1}^{m}}{f\left(V_{n+1}^{m}\right) V_{*}}\right)+\left(1-\frac{f\left(V_{*}\right)}{f\left(V_{n+1}^{m}\right)}\right)\left(\frac{f\left(V_{n+1}^{m}\right)}{f\left(V_{*}\right)}-\frac{V_{n+1}^{m}}{V_{*}}\right)\right] \\
& +\beta_{2} T_{*} g\left(I_{*}\right)\left[-\varphi\left(\frac{T_{*}}{T_{n+1}^{m}}\right)-\varphi\left(\frac{T_{n+1}^{m} g\left(I_{n}^{m}\right) W_{*}}{T_{*} g\left(I_{*}\right) W_{n+1}^{m}}\right)-\varphi\left(\frac{I_{*} W_{n+1}^{m}}{I_{n+1}^{m} W_{*}}\right)\right. \\
& \left.\left.-\varphi\left(\frac{g\left(I_{*}\right) I_{n+1}^{m}}{g\left(I_{n+1}^{m}\right) I_{*}}\right)+\left(1-\frac{g\left(I_{*}\right)}{g\left(I_{n+1}^{m}\right)}\right)\left(\frac{g\left(I_{n+1}^{m}\right)}{g\left(I_{*}\right)}-\frac{I_{n+1}^{m}}{I_{*}}\right)\right]\right\} \\
& -\sum_{m=0}^{N-1} \frac{D V_{*} \beta_{1} T_{*} f\left(V_{*}\right)}{k I_{*}(\Delta x)^{2}} \frac{\left(V_{n+1}^{m+1}-V_{n+1}^{m}\right)^{2}}{V_{n+1}^{m+1} V_{n+1}^{m}} .
\end{aligned}
$$

Similarly, we have

$$
\begin{aligned}
& \left(1-\frac{f\left(V_{*}\right)}{f\left(V_{n+1}^{m}\right)}\right)\left(\frac{f\left(V_{n+1}^{m}\right)}{f\left(V_{*}\right)}-\frac{V_{n+1}^{m}}{V_{*}}\right) \leq 0, \\
& \left(1-\frac{g\left(I_{*}\right)}{g\left(I_{n+1}^{m}\right)}\right)\left(\frac{g\left(I_{n+1}^{m}\right)}{g\left(I_{*}\right)}-\frac{I_{n+1}^{m}}{I_{*}}\right) \leq 0 .
\end{aligned}
$$

Thus, we get $\widetilde{G}_{n+1}-\widetilde{G}_{n} \leq 0$ for all $n \in \mathbb{N}$, that is, $\widetilde{G}_{n}$ is a decreasing sequence. Furthermore, since $G_{n}>0$, there is a limit $\lim _{n \rightarrow \infty} \widetilde{G}_{n} \geq 0$. Hence, $\lim _{n \rightarrow \infty}\left(\widetilde{G}_{n+1}-\widetilde{G}_{n}\right)=0$. Combined with system (8), we can show that $\lim _{n \rightarrow \infty} T_{n}^{m}=T_{*}, \lim _{n \rightarrow \infty} W_{n}^{m}=W_{*}, \lim _{n \rightarrow \infty} I_{n}^{m}=I_{*}$, and $\lim _{n \rightarrow \infty} V_{n}^{m}=V_{*}$ for all $m \in\{0,1, \ldots, N\}$, which implies that $E_{*}$ of system (8) is globally asymptotically stable. This completes the proof.

\section{Numerical simulations}

To illustrate our theoretical results obtained in the preceding sections, we carry out some numerical simulations in this section. To this end, we use two sets of system parameters that correspond to $\Re_{0}<1$ (when $E_{0}$ is globally asymptotically stable for both continuous and discretized models) and $\mathfrak{R}_{0}>1$ (when $E_{*}$ is globally asymptotically stable for both continuous and discretized models). For convenience, we consider system (3) with $f(V)=$ $V, g(I)=I$, and initial conditions

$$
T(x, 0)=100, \quad W(x, 0)=0.1, \quad I(x, 0)=0.1, \quad V(x, 0)=1, \quad x \in[0,10] .
$$

We first choose the parameters $\lambda=1, d=0.1, \beta_{1}=0.00025, \beta_{2}=0.00065, \delta=0.02, \gamma=$ $0.2, p=0.5, k=500, c=2.4$. It follows that $\mathfrak{R}_{0}=0.1065<1$. Theorem 3.2 implies that the infection-free equilibrium $E_{0}=(10,0,0,0)$ is globally asymptotically stable, which is numerically verified in Fig. 1.

Next, we set the parameters $\lambda=10, d=0.1, \beta_{1}=0.00025, \beta_{2}=0.00065, \delta=0.02, \gamma=0.2$, $p=0.5, k=500, c=2.4$. It follows that $\mathfrak{R}_{0}=9.5879>1$. By Theorem 3.3 the infection equilibrium $E_{*}=(10.4298,40.7137,16.2855,3392.8093)$ is globally asymptotically stable, as shown in Fig. 2. 

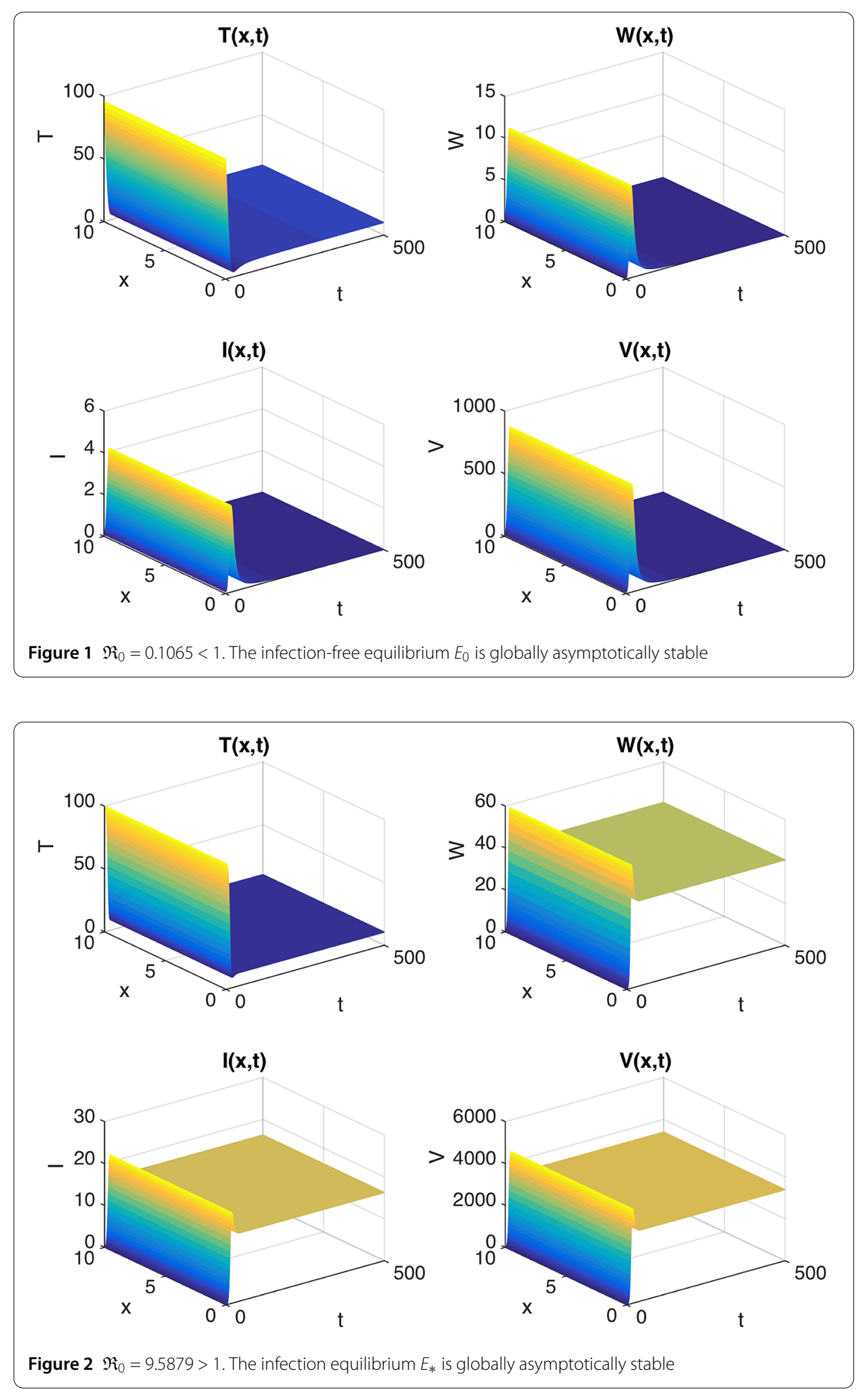

\section{Conclusions}

In this paper, we first investigate the global threshold dynamics of the equilibria of a whinhost virus infection model with both virus-to-cell and cell-to-cell transmissions. We then 
derive the discrete model by applying Mickens' nonstandard finite difference scheme. By using discrete analogue Lyapunov functions we show that the global stability of the equilibria of the discrete model is completely determined by the basic reproduction number $\mathfrak{R}_{0}$. If $\Re_{0} \leq 1$, then the infection-free equilibrium is globally asymptotically stable, whereas the infection equilibrium uniquely exists and is globally asymptotically stable when $\Re_{0}>1$. The results show that Mickens' discretization scheme can efficiently preserve the global asymptotic stability and positivity and boundedness of the solutions of the corresponding continuous model. Application of this method to a model with immune response or time delay is our future work.

\section{Acknowledgements}

This work was supported by the National Natural Science Foundation of China (\#11701445, \#11702214, \#11701451,

\#11501443) and Scientific Research Program Funded by Shaanxi Provincial Education Department (No. 17JK0787).

\section{Competing interests}

The authors declare that they have no competing interests.

\section{Authors' contributions}

All authors read and approved the final manuscript.

\section{Author details}

${ }^{1}$ School of Sciences, Xi'an University of Technology, Xi'an, P.R. China. ${ }^{2}$ Department of Mathematics, Northwest University, Xi'an, P.R. China. ${ }^{3}$ School of Mathematics and Statistics, Xi'an Jiaotong University, Xi'an, P.R. China.

\section{Publisher's Note}

Springer Nature remains neutral with regard to jurisdictional claims in published maps and institutional affiliations.

Received: 21 November 2017 Accepted: 15 March 2018 Published online: 27 March 2018

References

1. Nowak, M.A., Bangham, C.R.M.: Population dynamics of immune responses to persistent viruses. Science 272, 74-79 (1996)

2. Nowak, M.A., Bonhoeffer, S., Hill, A.M., Boehme, R., Thomas, H.C., McDade, H.: Viral dynamics in hepatitis B virus infection. Proc. Natl. Acad. Sci. 93, 4398-4402 (1996)

3. Korobeinikov, A.: Global properties of basic virus dynamics models. Bull. Math. Biol. 66, 879-883 (2004)

4. Funk, G.A., Jansen, V.A.A., Bonhoeffer, S., Killingback, T.: Spatial models of virus-immune dynamics. J. Theor. Biol. 233, 221-236 (2005)

5. Wang, K., Wang, W.: Propagation of HBV with spatial dependence. Math. Biosci. 210, 78-95 (2007)

6. Hattaf, K., Yousfi, N.: Global stability for reaction-diffusion equations in biology. Comput. Math. Appl. 66, 1488-1497 (2013)

7. McCluskey, C.C., Yang, Y.: Global stability of a diffusive virus dynamics model with general incidence function and time delay. Nonlinear Anal., Real World Appl. 25, 64-78 (2015)

8. Wang, F., Huang, Y., Zou, X.: Global dynamics of a PDE in-host viral model. Appl. Anal. 93, 2312-2329 (2014)

9. Xu, R., Ma, Z.: An HBV model with diffusion and time delay. J. Theor. Biol. 257, 499-509 (2009)

10. Dimitrov, D.S., Willey, R.L., Sato, H., Chang, L.J., Blumenthal, R., Martin, M.A.: Quantitation of human immunodeficiency virus type 1 infection kinetics. J. Virol. 67, 2182-2190 (1993)

11. Gummuluru, S., Kinsey, C.M., Emerman, M.: An in vitro rapid-turnover assay for human immunodeficiency virus type 1 replication selects for cell-to-cell spread of virus. J. Virol. 74, 10882-10891 (2000)

12. Bangham, C.R.M., Yang, Y., Zhang, T.H.: The immune control and cell-to-cell spread of human T-lymphotropic virus type 1. J. Gen. Virol. 84, 3177-3189 (2003)

13. Culshaw, R.V., Ruan, S., Webb, G.: A mathematical model of cell-to-cell spread of HIV-1 that includes a time delay. J. Math. Biol. 46, 425-444 (2003)

14. Sigal, A., Kim, J.T., Balazs, A.B., Dekel, E., Mayo, A., Milo, R., Baltimore, D.: Cell-to-cell spread of HIV permits ongoing replication despite antiretroviral therapy. Nature 477, 95-98 (2011)

15. Lai, X., Zou, X.: Modeling HIV-1 virus dynamics with both virus-to-cell infection and cell-to-cell transmission. SIAM J. Appl. Math. 74, 898-917 (2014)

16. Lai, X., Zou, X.: Modeling cell-to-cell spread of HIV-1 with logistic target cell growth. J. Math. Anal. Appl. 426, 563-584 (2015)

17. Pourbashash, H., Pilyugin, S.S., De Leenheer, P., McCluskey, C.: Global analysis of within host virus models with cell-to-cell viral transmission. Discrete Contin. Dyn. Syst., Ser. B 19, 3341-3357 (2014)

18. Yang, Y., Zou, L., Ruan, S.: Global dynamics of a delayed within-host viral infection model with both virus-to-cell and cell-to-cell transmissions. Math. Biosci. 270, 183-191 (2015)

19. Xu, J.H., Zhou, Y.: Bifurcation analysis of HIV-1 infectioin model with cell-to-cell transmission and immune response delay. Math. Biosci. Eng. 13, 343-367 (2016) 
20. Yang, Y., Zhou, J., Ma, X., Zhang, T.: Nonstandard finite difference scheme for a diffusive within-host virus dynamics model with both virus-to-cell and cell-to-cell transmissions. Comput. Math. Appl. 72, 1013-1020 (2016)

21. Wang, T., Hu, Z., Liao, F., Ma, W.: Global stability analysis for delayed virus infection model with general incidence rate and humoral immunity. Math. Comput. Simul. 89, 13-22 (2013)

22. Sigdel, P.P., McCluskey, C.C.: Global stability for an SEl model of infectious disease with immigration. Appl. Math. Comput. 243, 684-689 (2014)

23. Abdelmalek, S., Bendoukha, S.: Global asymptotic stability of a diffusive SVIR epidemic model with immigration of individuals. Electron. J. Differ. Equ. 2016, Article ID 284 (2016)

24. Li, J., Ma, Z., Brauer, F.: Global analysis of discrete-time SI and SIS epidemic models. Math. Biosci. Eng. 4, 699-710 (2007)

25. $\mathrm{Hu}$, Z., Teng, Z., Zhang, L.: Stability and bifurcation analysis in a discrete SIR epidemic model. Math. Comput. Simul. 97, $80-93$ (2014)

26. Zhou, Y., Ma, Z., Brauer, F.: A discrete epidemic model for SARS transmission and control in China. Math. Comput. Model. 40, 1491-1506 (2004)

27. Mickens, R.E.: A SIR-model with square-root dynamics: an NSFD scheme. J. Differ. Equ. Appl. 16, 209-216 (2010)

28. Ma, X., Zhou, Y., Cao, H.: Global stability of the endemic equilibrium of a discrete SIR epidemic model. Adv. Differ. Equ. 2013, Article ID 42 (2013)

29. Mickens, R.E.: Nonstandard Finite Difference Models of Differential Equations. World Scientific, Singapore (1994)

30. Mickens, R.E.: Discretizations of nonlinear differential equations using explicit nonstandard methods. J. Comput. Appl. Math. 110, 181-185 (1999)

31. Mickens, R.E.: Nonstandard finite difference schemes for differential equations. J. Differ. Equ. Appl. 8, 823-847 (2002)

32. Sekiguchi, M.: Permanence of a discrete SIRS epidemic model with time delays. Appl. Math. Lett. 23, 1280-1285 (2010)

33. Sekiguchi, M., Ishiwata, E.: Global dynamics of a discretized SIRS epidemic model with time delay. J. Math. Anal. Appl. 371, 195-202 (2010)

34. Korpusik, A.: A nonstandard finite difference scheme for a basic model of cellular immune response to viral infection. Commun. Nonlinear Sci. Numer. Simul. 43, 369-384 (2017)

35. Ding, D., Ma, Q., Ding, X.: A non-standard finite difference scheme for an epidemic model with vaccination. J. Differ. Equ. Appl. 19, 179-190 (2013)

36. Enatsu, Y., Nakata, Y., Muroya, Y., Izzo, G., Vecchio, A.: Global dynamics of difference equations for SIR epidemic models with a class of nonlinear incidence rates. J. Differ. Equ. Appl. 18, 1163-1181 (2012)

37. Yang, Y., Ma, X., Li, Y.: Global stability of a discrete virus dynamics model with Holling type-Il infection function. Math. Methods Appl. Sci. 39, 2078-2082 (2016)

38. Qin, W., Wang, L., Ding, X.: A non-standard finite difference method for a hepatitis B virus infection model with spatial diffusion. J. Differ. Equ. Appl. 20, 1641-1651 (2014)

39. Pazzy, A.: Semigroups of Linear Operators and Applications to Partial Differential Equations. Springer, New York (1983)

40. Smoller, J.: Shock Waves and Reaction-Diffusion Equations. Springer, Berlin (1983)

41. Protter, M.H., Weinberger, H.F.: Maximum Principles in Differential Equations. Prentice Hall, Englewood Cliffs (1967)

42. Henry, D.: Geometric Theory of Semilinear Parabolic Equations. Springer, Berlin (1993)

\section{Submit your manuscript to a SpringerOpen ${ }^{\circ}$ journal and benefit from:}

- Convenient online submission

- Rigorous peer review

Open access: articles freely available online

- High visibility within the field

Retaining the copyright to your article

Submit your next manuscript at $\gg$ springeropen.com 